\title{
Penser l'Étranger
}

L'assimilation dans les représentations sociales et les théories sociologiques de l'immigration

Jocelyne Streiff-Fénart

\section{(2) OpenEdition}

1 Journals

Édition électronique

URL : http://journals.openedition.org/ress/2339

DOI : $10.4000 /$ ress.2339

ISSN : 1663-4446

Éditeur

Librairie Droz

\section{Édition imprimée}

Date de publication : 1 juin 2013

Pagination : 65-93

ISBN : 978-2-600-01749-7

ISSN : 0048-8046

Référence électronique

Jocelyne Streiff-Fénart, "Penser l'Étranger », Revue européenne des sciences sociales [En ligne], 51-1 |

2013, mis en ligne le 01 janvier 2017, consulté le 21 décembre 2020. URL : http://

journals.openedition.org/ress/2339; DOI : https://doi.org/10.4000/ress.2339 


\title{
PENSER L'ÉTRANGER \\ L'ASSIMILATION DANS LES REPRÉSENTATIONS SOCIALES ET LES THÉORIES SOCIOLOGIQUES DE L'IMMIGRATION
}

JOCELYNE STREIFF-FÉNART

CNRS / Université de Nice Sophia-Antipolis-URMIS

streiff@unice.fr

Résumé. Cet article retrace la genèse de la notion d'assimilation en montrant comment elle a fonctionné, aux États-Unis et en Europe, comme une matrice cognitive conduisant à penser la migration comme entrée d'étrangers dans la Nation. On examine ensuite les modalités selon lesquelles se recomposent actuellement les frontières entre des «nous》 et des «eux» dans la société française, en se demandant dans quelle mesure les références accrues aux distinctions ethniques et raciales depuis les années 2000 signalent un abandon du paradigme de l'assimilation. On évoque en conclusion des pistes de recherche qui, au delà de la désaffection qui s'attache au mot «assimilation» ou des tentatives d'en renouveler l'idée, en minent de façon radicale les présupposés.

Mots-clés: assimilation, sociologie de l'immigration, ethnicité, race, nation.

\begin{abstract}
This article traces the history of the notion of assimilation in the US and Europe. I argue that it has worked as a cognitive scheme leading to grasp migration as the entry of foreigners into the Nation. I then examine the making of new boundaries in French society that emphasize categories of race and ethnicity to set apart French citizens from foreign origins. I wonder if the focus on ethnic and racial distinctions either expresses the dropping of the assimilation paradigm, or allows its preservation by adjusting its meaning to the new configuration. I then point to some research viewpoints that radically challenge the assumptions that underly the assimilationist framework.
\end{abstract}

Keywords: assimilation, migration, ethnicity, race, nation. 
La sociologie de l'immigration est une science des métaphores, métaphore marine des vagues, des flux, des courants, métaphore agricole de la transplantation, de la greffe, de l'enracinement, métaphore biologique de l'assimilation et de l'absorption.

Parmi toutes ces images et analogies qui figurent le rapport entre le migrant et la société réceptrice, celle de l'assimilation qui représente la société comme un corps vivant humain et l'immigré comme un corps étranger à digérer, est sans nul doute la mieux partagée. Catégorie à la fois politique et analytique, téléologique et descriptive, elle a circulé entre les différents points du globe où s'expérimentent les modèles d'intégration des immigrés (France, Grande Bretagne, Belgique, États-Unis, Israël, Australie, etc.) mais aussi entre les agents du pouvoir politique (administrateurs coloniaux, hauts fonctionnaires), les organes de fabrication et de diffusion des «débats de société» (presse, émissions radiophoniques, reportages télévisés) et les cercles savants (universitaires, experts).

Enracinée dans la tradition sociologique de l'organicisme, la métaphore de l'assimilation doit aussi beaucoup à une vision «progressiste » du changement et dela diversité humaine : le même chemin parcouru par l'humanité sur une échelle temporelle du progrès peut être parcouru en accéléré par la rencontre entre des groupes humains situés à des stades différents d'évolution. Cette conception évolutionniste qui vise à intégrer les autres en les faisant accéder à l'universel est présente dans l'espace intellectuel français depuis la Révolution française où elle a constitué l’arrière plan de la notion de régénération des peuples soumis. Une autre métaphore, de source américaine, a accentué à travers l'image du creuset le sens du mélange et de la fusion plutôt que celui de l'absorption, ces deux faces de l'assimilation se trouvant représentées dans l'histoire des États-Unis par les idéologies fusionnelles ou exclusivistes du melting-pot et de l'anglo-conformity.

Bien que des voix s'élèvent périodiquement pour déplorer la confusion entre ses usages politiques et normatifs et sa définition comme concept opératoire, la sociologie de l'immigration n'en a pas encore fini avec la notion d'assimilation et constate régulièrement son retour (voir par exemple Brubaker, 200I). 
On se propose dans cet article d'interroger la longévité d'une notion pourtant floue et ambiguë, en commençant par interroger cette ambiguïté elle-même, puis en examinant ses usages et reformulations dans les sciences sociales aux États-Unis et en France. On abordera ensuite les modalités selon lesquelles se recomposent actuellement les frontières entre des «nous» et des «eux» dans la société française, en se demandant dans quelle mesure les références accrues aux distinctions ethniques et raciales depuis les années 2000 signalent un abandon du paradigme de l'assimilation.

\section{L' ASSIMILATION Une notion fondamentalement ambiguë et contradictoire}

Une première source d’ambiguïté particulièrement visible dans l'acception française de la notion est qu'elle entretient la confusion entre les dimensions juridique, biologique et culturelle de la différence entre les immigrés et les membres de la société à laquelle ils sont appelés à s’assimiler.

On peut voir, notamment, qu'alors même que l'assimilation est indissociable d'une conception individuelle et contractuelle de la citoyenneté ${ }^{1}$ l'acte juridique qui fait de l'étranger un national (la naturalisation) consacre la subordination des catégories juridiques (national / étranger) aux catégories culturelles. Le droit français fait en effet de l'assimilation culturelle de l'immigré (attestée par son adhésion aux valeurs et sa connaissance de l'histoire et de la culture françaises) une condition de la naturalisation (loi de 1927 sur la nationalité, ordonnance de 1945 portant Code de la Nationalité). L'idée d'une acquisition de la culture par le processus de socialisation est tout à fait congruente avec la conception évolutionniste et progressiste dont on a souligné plus haut qu'elle constitue l'arrière plan de la métaphore de l'assimilation. La contradiction réside dans sa coexistence avec une autre métaphore biologique très présente dans les sciences sociales: celle de l'hérédité, qui naturalise les différences de culture par la différence dite d'origine et les rapporte à des groupes ethnique-

I Celle propre à la représentation de la Nation comme corps de citoyens liés par la volonté de vivre ensemble qui de Rousseau à Renan, s'est construite par opposition aux contre-modèles de la conception anglaise de la citoyenneté et à la Nation ethnique allemande. 
ment ou nationalement référencés. Dun côté, les valeurs auxquelles sont tenus d’adhérer contractuellement les candidats à la naturalisation (la laïcité, l'égalité entre hommes et femmes) sont supposées d'emblée acquises par les Français de naissance, c'est à dire ceux dont les ancêtres (en remontant à un nombre indéterminé de générations) ont contribué à écrire le récit national. De l’autre côté, les immigrés sont supposés dépositaires de cultures (le plus souvent réifiées sous formes de traits culturels décontextualisés, comme l’endogamie ou le «patriarcat») qui leur appartiendraient en propre et qui expliqueraient leurs comportements et ceux de leurs enfants. ${ }^{2}$

Le principe d'assimilation porte donc en lui-même sa propre contradiction puisque voulant mettre l'accent sur la culture contre la théorie héréditariste de la race, il retrouve cette dernière sous la forme euphémisée de l’ethnie dans sa définition la plus traditionnelle de groupe fondé sur une ascendance commune et porteur de culture transmise de génération en génération.

Cette contradiction, consubstantielle à la notion française d'assimilation permet, comme l’a rappelé Jean-Loup Amselle (1996), de faire co-exister une conception atomistique de la Nation (la communauté des individus citoyens) et le postulat de l'existence d'une pluralité de groupes présents en son sein, que ceux-ci soient pensés en termes raciaux ou ethniques. Elle entraîne une autre contradiction: la même notion qui affirme en théorie l'accès de tout individu à l'universel est utilisée en pratique comme un outil de sélection et de tri entre des populations inégalement dotées de capacités à s'assimiler.

C'est en effet en faisant fond sur cette dualité universalité / pluralité que la notion d’assimilation a pu être utilisée alternativement selon les périodes et les objectifs politiques pour désigner le processus d’accession des peuples «inférieurs » à la civilisation, la transformation des enfants de paysans (ou d'immigrés) en Français grâce à l'école républicaine, mais aussi comme un principe de division entre populations assimilables et non assimilables, ces dernières étant porteuses d'une différence essentielle non convertible (une «race», donc). Déjà

2 Cette vision s'affirme nettement dans les premiers rapports (1991, 1995) du Haut Conseil à l'Intégration. Pour une formulation récente de l'approche «culturaliste» dans le champ académique, voir Lagrange, 2010. 
présente dans les débats sur l'esclavage à la Constituante de I79I pour distinguer les Métis et les Noirs, la distinction entre assimilables et non assimilables est mise en œuvre dans l'entre deux guerre pour trier les populations relevant d'une immigration de peuplement (Italiens, Belges, Suisses) et celles (Africains, Levantins et Asiatiques) relevant d'une immigration de travail ayant vocation à repartir (Schor, 1996). L’idée de prédispositions plus ou moins grandes à l’assimilation selon les «cultures d'origine» des groupes a été relayée et confortée par les instances scientifiques. Elle constitue l’arrière plan des grandes enquêtes sur l'assimilation des immigrés menées depuis les années 1950 jusqu'à nos jours. Commentant les résultats de l'enquête menée en Belgique par l'équipe de René Clémens, Alain Girard (1954) souligne que «la capacité d’assimilation des Polonais apparaît moins vive que celles des Italiens parce que la "distance culturelle” qui les sépare des Belges est beaucoup plus grande» (p. I48). L’enquête MGIS (Mobilité Géographique et Insertion Sociale) menée par l'INED et l'INSEE dans les années 1990 s'attache à mesurer le maintien ou la déperdition de comportements culturels spécifiques (notamment matrimoniaux et religieux) attribués à des populations construites comme des catégories ethniques («arabes algériens », «berbères » ou «kabyles», «turcs », «kurdes», «mandés d’Afrique noire», etc.). Cette enquête sur laquelle on reviendra illustre de façon exemplaire la façon dont la contradiction propre à l'assimilation peut être exposée sans être aperçue puisque on peut lire sous la plume du Président du HCI qui préface l'ouvrage qui en est tiré (Tribalat, 1995) que «ces recherches (fondées donc sur la comparaison de groupes ethniques) renforcent notre attachement à l'exemple français quelquefois mis en doute et notre conviction en sa valeur de modèle » (sous entendu assimilationniste contre l'exemple anglo-saxon multi-culturaliste).

Une autre ambiguïé constante dans les usages de la notion est qu'elle peut renvoyer à une action transitive (assimiler) ou réfléchie (s'assimiler) selon le référent dont elle est le prédicat. Lorsque elle est pensée comme une tâche dévolue à l'État dans le cadre de l'unification nationale (assimilation des Juifs et des habitants des «terroirs») ou de la mission civilisatrice coloniale, l'assimilation est une vertu (le «génie assimilateur», «la puissance assimilatrice») qui manifeste la capacité de l'État à ranger sous son ordre juridique et social 
des populations qui lui étaient étrangères. Lorsqu’elle a pour référent l'individu appelé à s'assimiler, elle se présente comme une épreuve (un examen) attestant que l'immigrant a passé avec succès le test le qualifiant comme faisant partie du Nous national. Le privilège accordé à la culture contre la race trouve ici son plein rendement puisque, contrairement à la race qui s'impose au sujet comme un stigmate, la culture qui relève de l'apprentissage suppose de sa part un travail d'acquisition et une disposition au changement.

C'est cette dimension normative, qui lie étroitement la «bonne volonté » culturelle et la loyauté à la Nation qu'on retrouve dans les procédures de naturalisation. Comme le montre Abdellali Hajjat (20ı), l'évaluation du «défaut d'assimilation » pouvant motiver le rejet de la demande n'obéit pas à des critères précis, mais repose pour une large part sur l'appréciation individuelle des agents : s'agissant par exemple du couvre-chef des femmes musulmanes, on attend d'eux qu'ils détectent la véritable signification d'un comportement permettant de conclure à son caractère anodin (traditionnel) ou potentiellement dangereux (islamiste).

Le classement des comportements culturels selon une double grille civilisation/dangerosité n’est pas sans évoquer celui qui, au lendemain de la Première Guerre mondiale, conduisait à distinguer deux catégories de migrants inassimilables, ceux qui font partie des «races inférieures» (les Indigènes des colonies) et ceux qui appartiennent aux «races antagonistes» (les Allemands) (Noiriel, 2007, p. I2), mais la menace se cristallise désormais sur les offenses à la laïcité plus que sur la trahison à la patrie. La notion moderne de «communautarisme » évoquée de façon récurrente à propos des immigrés provenant de pays musulmans concentre à elle seule les deux significations (défaut de modernité + menace de constitution de «noyaux étrangers »), tandis que la question du voile féminin analysée par Hajjat les distingue comme deux traits comportementaux, renvoyant soit à l'imposition d'une tradition de soumission de la femme héritée des cultures patriarcales, soit à un acte volontaire de défi à la République. 


\section{L'IMMIGRÉ COMME ÉTRANGER À LA NATION}

Bien que la France soit souvent considérée comme le pays par excellence du modèle assimilationniste, l'idiome de l'assimilation, relève Françoise Lorcerie, n'est pas typiquement français, il est typiquement national. «L'assimilation ne peut se passer d'un foyer qui la polarise. Ce foyer c'est la Nation» (Lorcerie, 1999, p. 322). Le lien entre assimilation et Nation s'est toutefois noué selon des modalités spécifiques dans les différents pays d'immigration et les sciences sociales y ont diversement contribué.

Aux États-Unis, où formation de la Nation et immigration sont allées de pair, la sociologie naissante s'est emparée dès le début du $\mathrm{xx}^{\mathrm{e}}$ siècle des questions relatives aux modes d'entrée des immigrants dans leur nouvel environnement et en a fait un thème d'étude prioritaire. Selon Andreas Wimmer et Nina Glick Schiller (2003), c'est entre autres l'intérêt précoce porté par la sociologie à l'assimilation des immigrants qui a contribué à l'inscrire dans une perspective qu'ils dénoncent comme un «nationalisme méthodologique»:

Scholars who share this intellectual orientation assume that countries are the natural units for comparative studies, equate society with the nation state, and conflate national interests with the purposes of social sciences. (p. 276).

Cette naturalisation de la forme de l'État-nation par les sciences sociales se trouve déjà selon eux dans les écrits des fondateurs de l'école de Chicago. Il faut toutefois regarder les choses de très loin pour leur attribuer une telle responsabilité et leur position apparaît plus complexe que ne veulent bien le dire les auteurs. Pour une grande partie, les premiers écrits de l'école de Chicago relèvent d'une sociologie des interactions et des transactions qui ont pour cadre la ville plus que la Nation.

L'immigrant polonais de William Thomas et Florian Znaniecki est avant tout un «paysan» dont le nouveau self en immigration se forme dans le processus de désorganisation des groupes primaires de la famille et de la communauté, du délitement des normes sociales qu'ils transmettaient, et de la réorganisation des fragments épars de cette organisation locale ancienne selon des formes d'organisation (l'association volontaire) propres aux sociétés urbaines modernes. 
Les auteurs récusent d'ailleurs explicitement une vision «nationaliste» qui concevrait l'assimilation comme l'entrée dans (ou la sortie d') un groupe national :

In fact, if we look at the Poles in America not from the standpoint of Polish or American national interests but from that of an objective sociological inquiry, we find that the problem of individual assimilation is at present an entirely secondary and unimportant issue. (Thomas et Znaniecki, 1918-1920, vol. 5, viii).

C'est non pas à la nation américaine mais à la société américano-polonaise qui se forme dans l'immigration avec ses propres normes et institutions, que l'immigrant polonais doit s'ajuster.

La ville constitue le cadre d'analyse de l'assimilation pour les immigrants venus d'Europe comme pour les Noirs provenant du Sud rural. Dans l'analyse que faisait Charles Johnson de l'adaptation du Noir à la société urbaine de Chicago, les obstacles à l'assimilation ne sont pas de nature « raciale » ni «culturelle», au sens d'une tradition, mais de l'ordre de troubles dans l'interaction :

The great majority of migrants are laborers. Many of them are ignorant and rough mannered, entirely unfamiliar with standards of conduct in modern cities [... They felt strange and uncertain as to how they should act. Many whites and Negro long resident in Chicago have said that they could tell a migration Negro by his ill-at-ease manner and often by his clothes [...] Few white people realized how uncertain the southern Negro felt about making use of his new privilege of sitting anywherere in the car, instead of being «Jim crowed». (Johnson, 1922, p. 30I).

Et s'il est vrai que le race relation cycle de Park (1926) avec ses différentes phases - concurrence, conflit, compromis, assimilation - a une forte dimension normative (en ce sens que l'assimilation comme processus d'interpénétration et de fusion y apparaît comme la fin à la fois inéluctable et désirable du cycle), la Nation n'y est pas donnée comme un cadre préalable à des groupes étrangers appelés à s'y intégrer, mais comme l'horizon qui donne sens à la solidarité culturelle entre tous les groupes qui composent la société américaine. La métaphore de l'Amérique comme un creuset où se forge l'homme nouveau à partir de la fusion des peuples, popularisée en I9ı par la pièce de théâtre d’Israël Zangwill, The melting Pot, n'est pas très loin. 
Il est toutefois indéniable que c'est en s'appuyant en partie sur les concepts forgés par les chercheurs de Chicago que la sociologie des migrations de l'époque ultérieure a pleinement développé ce que Wimmer et Glick Schiller (1992) appellent le «container model of society», opérant une coupure analytique entre les systèmes de relations qui se mettent en place à l'intérieur des frontières et les réseaux de relations que les migrants entretiennent à l'extérieur. Sous-tendues par les postulats fonctionnalistes en vigueur à cette époque, les recherches sur les migrations menées dans les années 1950-1960 sont fondées sur l'analyse du rapport dualiste entre une société conçue comme une totalité homogène et des groupes culturellement différents dont le comportement menace l'équilibre et la cohésion du système. Une multitude de travaux vont s'attacher au cours de ces années à étudier le processus d'ajustement entre l'acculturation des nouveaux arrivants et leur acceptation par la société d'accueil (Warner et Srole, 1945 ; Borie, 1959; Gordon, 1964). Contre cette pensée dominante vont s'élever dans les années 1970 les chercheurs qui, constatant à la suite de Nathan Glazer et Daniel Patrick Moynihan (1963) le maintien des liens ethniques dans la société américaine, vont promouvoir le concept d'ethnicity et en faire une catégorie générale de la vie sociale.

En Europe, lorsque la science sociale se saisit de la question des migrations à la fin du XIX ${ }^{e}$ siècle, c'est le rapport entre villes et campagnes plutôt qu'entre nations qui fournit le cadre d'analyse des mouvements de populations. Les premières études réellement scientifiques sur les migrations, conduites par des géographes, visaient à établir des régularités dans le déplacement des hommes et les facteurs qui déterminent le caractère attractif ou répulsif de différentes aires géographiques. Il n'était pas nécessaire alors de traverser une frontière pour être considéré comme un migrant. Pour penser la migration, on s'intéressait plus aux flux migratoires qu'aux stocks d'immigrants (pour utiliser des notions modernes) et la proximité ou la distance géographiques semblaient plus pertinentes que la proximité ou la distance culturelles. Lorsqu'il formule ses lois sur la migration, Ernst Georges Ravenstein (1889) cherche à établir les causes de la mobilité qu'elle soit interne ou internationale et s'il utilise la notion d'absorption, ce n'est pas pour décrire l'aboutissement du processus 
d'assimilation, comme le feront les auteurs ultérieurs, mais pour préfigurer, en opposition avec celle de dispersion, ce qu'on identifiera plus tard comme des facteurs push and pull entre zones d'émigration et d'immigration.

L'émergence de la notion d'assimilation est liée au changement de paradigme qui fait de la Nation le cadre de l'accueil des immigrants pensé comme une relation hôte / invité, cadre qui sera développé de la façon la plus explicite par Sheila Patterson dans son enquête sur les Jamaïcains de Brixton, menée dans les années 1950. Comme tous les chercheurs britanniques de sa génération, Patterson envisage la situation de ces nouveaux immigrés dans le cadre des «relations raciales» et utilise pour les désigner des termes de race (colored, black, negros). Mais le recadrage de la situation migratoire comme relation d'hospitalité veut précisément, dans une période marquée par les émeutes raciales, mettre l'accent sur les comportements et les traits culturels susceptibles de se modifier au cours du temps plus que sur la marque raciale. L'utilisation d'un classique modèle en succession de phases inspiré par l'école de Chicago lui permet de déplacer la situation des black strangers d'une problématique du conflit racial vers une problématique de l'adaptation, laissant entrevoir la possibilité d'une assimilation future (Patterson, 1963). ${ }^{3}$

En France, c'est comme doctrine juridique et outil de gestion de la force de travail plutôt que comme concept scientifique que la notion d'assimilation va s'imposer. L’embauche massive de main d'œuvre étrangère après la première guerre mondiale focalise l'attention sur les critères de sélection des nouveaux venus en vue de correspondre au mieux aux besoins économiques et d'éviter la formation de «noyaux allogènes» dangereux sur le territoire national. (Schor, p. 76). Dans la France de l'entre deux guerres, tout un corps de hauts fonctionnaires et de spécialistes (juristes, géographes, médecins) va s’atteler à faire le tri entre les populations indésirables et celles prédisposées à s'assimiler (Paon, 1926; Mauco, 1932). Avec la mobilisation intensive de la force de travail des années 1960, la question de l'assimilation prend la forme de

3 La relation hôte/invité est par contre évoquée, en Allemagne, par le terme gastarbeiter qui officialise dans la dénomination la mise à distance de la Nation des immigrés longtemps considérés en dépit de l'installation des familles, comme des «oiseaux de passage». 
«l'adaptation au travail industriel» et le verbe «assimiler » retrouve, mais avec un autre sens, sa forme transitive: on ne demande pas à l'ouvrier immigré de s'assimiler mais d'assimiler (comme on demande à un élève d'assimiler une leçon) les exigences de la vie au travail dans le milieu de la grande industrie (Descloitres, 1967). Mais l'immigré a beau être considéré comme une pure force de travail, la classification des «ethnies » entre assimilables et non assimilables reste toutefois de règle. On y retrouve la hiérarchie qui place les occidentaux au sommet et les non occidentaux à la base, même si les groupes inclus dans ces catégories ont changé, les Espagnols et les Portugais prenant la place des Belges et Italiens d'antan, tandis que les «Africains» - pouvant selon les cas inclure ou exclure leur composante maghrébine -, se trouvent toujours tout en bas de l'échelle. Un rapport interne de la Régie Renault fait ainsi mention de «la faible adaptabilité du personnel d'origine africaine, en particulier d'Afrique noire, et pour les mêmes ethnies, la faible propension à assimiler la société française ». (RNUR, 1976, cit. in Perriaux et Varro, 199I).

Lorsque les sciences sociales se saisissent de la question migratoire à partir des années 1970, l'élaboration théorique de la notion d'assimilation dans l'espace académique nord-américain sera peu commentée. Les problématiques sont plutôt inspirées par des perspectives marxistes appréhendant l'immigration comme le produit d'une division internationale du travail (Meillassoux, 1976; Mercier, 1977) ou par la sociologie de la domination de l'école de Bourdieu qui construit la migration comme un fait social total exprimant les rapports de subordination entre les sociétés du Nord et du Sud (Sayad, 198I). Un autre courant a abordé les migrations sous l'angle des relations interethniques en milieu urbain (Guillon et Taboada-Leonetti, I986; De Rudder, 1987 ; Borgogno, 1988) ou des négociations intergénérationnelles des identités (Oriol, 1984; Hovanessian, 1992 ; Streiff-Fénart 1994). Dans tous les cas, la formulation de la question migratoire dans la sphère académique s'est d'emblée inscrite dans une sociologie critique prenant ses distances avec 
les idéologies nationales de l'intégration ou a déplacé la question vers celle de l'intégration dans la classe ouvrière (Granotier, 1970; Tripier, 1987). ${ }^{4}$

La notion d’assimilation s'est trouvée, quant à elle, largement utilisée jusqu’à aujourd'hui par les démographes (Girard et Stoezel, 1953-1954; Girard 1954; Tribalat, 1995; Todd, 1994)..$^{5}$ Il n'est donc guère étonnant que cette discipline ait fourni l'arène dans laquelle ont fini par se manifester au début des années I990 les contradictions de la notion relevées plus haut.

La prise en compte de l'origine ethnique dans l'enquête MGIS a donné lieu à une controverse dont Adrian Favell (200I) a relevé le caractère «ironique » puisqu'elle revient à présenter comme un destructeur de tabous un rapport «aussi traditionnel que peut l'être une étude française » sur l'assimilation, mot qui se trouve réhabilité dans cette enquête contre les formulations plus neutres «d’intégration » ou «d'insertion » préférées depuis plusieurs années dans les rapports officiels.

L’innovation est toutefois bien réelle, mais elle se situe moins dans le cadre analytique de l'étude (qui reste en effet fermement ancrée dans le «nationalisme méthodologique ») que dans le changement radical, allant sans doute au-delà des intentions des responsables de l'enquête, de la grammaire des catégories à travers lesquelles on pense le rapport social induit par l'immigration.

En prenant l'origine plutôt que la nationalité comme variable, ce n'est pas seulement l'extension de la classe opposée à «Français » qui se trouve redéfinie, mais également la compréhension de l'ensemble «Français » qu’on lui oppose. Pour se différencier de l'étranger devenu un «ethnique », le Français doit être nommé d’un terme également ethnique. C’est la fonction que remplit l’adjonc-

4 Au cours de la même décennie, vont se multiplier parallèlement les travaux empiriques sur les différents aspects de l'intégration commandités par les ministères (du Travail, de l'Éducation, de la Famille) en charge de la question et donnant lieu à de nombreux rapports d'études.

5 La participation à l'enquête de l'INED de 1953 d'un jeune chercheur, Léo Bogart, formé à Chicago n'aura guère d'influence. Lui-même distingue nettement son propre intérêt scientifique qui, dans la droite ligne de l'école de Chicago le porte à étudier «la recréation d'un ordre social chez les Nord-Africains en France» du souci de l'INED d'identifier «les causes socio-psychologiques qui sont derrière une tendance vers ou contre l'adaptation et l'assimilation» (cité in Escafré-Dublet, 201I). 
tion de la qualification «de souche » qui indique la réduction de «Français » au niveau, infra-national, de l'ethnicité.

Bien que cet aspect soit resté très généralement inaperçu, l'un des effets de l'introduction de données relatives à l'origine des immigrés est de faire à rebours le chemin décrit par les Hughes dans les années 1950 à propos de la sociologie américaine: auparavant, remarquaient-il, tous les humains (à part les «hommes marginaux») étaient supposés appartenir à des groupes ethniques. La nouvelle conception minus one des groupes ethniques revient à ne désigner comme ethniques que les groupes qui diffèrent du groupe majoritaire qui est lui, soustrait à l'ethnicité (Hughes et Hughes, 1952).

En réintroduisant le majoritaire dans la catégorie de l'ethnicité (dont «Français de souche» devient une classe à côté de Mandé, algérien, kabyle, turc, etc.), l'enquête MGIS en vient ainsi, en contradiction avec ses propres objectifs, à miner la fiction républicaine qui pour faire de la francité une identité politique potentiellement universelle suppose l'existence d'un majoritaire dont la culture reste en creux.

\section{FRONTIÈRES ET DÉNOMINATIONS}

La pluralité des groupes dans la société américaine a été pensée par un jeu d'opposition entre catégories raciales et catégories ethniques. Des processus d’amalgamation / différenciation ont constamment redéfini les frontières de la race et de l'ethnie au sein de la Nation et redistribué les groupes dans les catégories en fonction des trajectoires de mobilité et des luttes sociales pour la requalification: fragmentation des immigrés entre les nouveaux venus, allemands ou scandinaves, pouvant rejoindre la population mainstream et ceux, européens catholiques, italiens et irlandais, grecs ou juifs d'Europe orientale, à qui était déniée la pleine appartenance au groupe Blanc; amalgamation des identités locales (Siciliens ou Piémontais) ou nationales (Chinois, Japonais) dans de nouvelles identités englobantes (Italiens, Asiatiques) ; création d'une catégorie intermédiaire de groupes blancs (les hyphenated-groups) distincte des 
WASP $^{6}$ et des Afro-américains ; pleine reconnaissance des anciennes immigrations européennes du Sud et orientales dans la catégorie des Américains Blancs refoulant vers la catégorie des minorités de couleur les nouveaux migrants centre américains ou caribéens, etc. Tous ces processus de changement des frontières ethnico-raciales ont été abondamment décrits et analysés dans la littérature américaine, aussi bien par les auteurs qui proposent des paradigmes alternatifs à l'assimilation que par ceux qui tentent d'en renouveler les termes (Horowitz, 1975 ; Glazer et Moynihan, 1975 ; Zollberg et Woon, 1999 ; Alba et Nee, 2003; Roediger, 199I ; Wimmer, 2008).

Le processus d'incorporation des populations migrantes a suivi en France des chemins tout différents. L’«idiome de la Nation assimilationniste » qui est propre à la France (Brubaker, 1997) a instauré et maintenu une opposition binaire entre outsiders et insiders autour d'une double clôture des frontières territoriales de l'État et culturelles de la Nation, le franchissement de la frontière (l’assimilation) laissant inchangée cette dualité.

Mais si la catégorie de Français qui forme le socle de cette partition est restée jusqu'à une date récente une entité non discutable, la catégorie qui lui fait face dans cette logique binaire y a constamment été l'objet de différentiations en fonction des enjeux migratoires de l'époque.

Ces redéfinitions se manifestent en particulier dans l'évolution des termes (ou des changements de significations attachées à un même terme) qui désignent les populations cibles des politiques publiques, et dans les labels alternatifs que tentent de leur opposer les groupes ainsi désignés. Les premiers, qui sont dotés de la force performative conférée par les identifications étatiques, constituent des opérations de désignation par lesquelles se construit le sens politique de l'immigration, tandis que les seconds supposent l'émergence d’acteurs collectifs susceptibles de résister à ces impositions et de mettre en circulation des auto-identifications alternatives. de caste. 
Gérard Noiriel (2007) rappelle que le terme immigré a d’abord été une appellation créée en 1920 par le PCF (Main d’Oeuvre Immigrée: MOI) préférée à la désignation comme étranger utilisée dans les textes officiels. Actuellement ce terme s'applique dans les nomenclatures françaises (INSEE, INED) aux plus étrangers de tous les étrangers : les personnes nées étrangères à l'étranger (mais pas aux personnes qui ont effectivement migré tout en ayant la nationalité française). Dans le langage courant, l'usage du terme immigré est encore plus restrictif puisqu’il exclut très généralement de la catégorie, les étrangers nés à l'étranger provenant du premier monde (les Américains, les Suisses ou les Japonais). Si l'étranger est convertible en français par acquisition de la nationalité française, l'immigré reste tel tout au long de sa vie dans les taxinomies officielles, et l'on peut souvent constater que, au delà de sa propre biographie, son «immigritude » se transmet à ses enfants dans les désignations courantes («quartiers immigrés », «enfants immigrés » dans l’école, «jeunesse immigrée », etc.).

À partir des années 1960 et pendant une vingtaine d’années, l'immigré ne pourra se concevoir autrement qu'en «travailleur». La logique démographique ayant définitivement cédé le pas à une logique de main d'œuvre, c'est même l'étranger en général qui est saisi comme tel. La revue Esprit qui a consacré un numéro spécial aux «Étrangers en France» en I966 propose en 1973 un numéro spécial sur «Les travailleurs étrangers en France». La définition de l'étranger ou de l'immigré comme travailleur fait consensus dans les travaux académiques et les rapports administratifs ${ }^{7}$, mais aussi dans les associations de soutien aux immigrés comme le GISTI (Groupe d'information et de soutien des travailleurs immigrés) ${ }^{8}$ et dans les mouvements militants qui emblématisent la figure de l'OS comme couche inférieure et surexploitée du salariat. Même

7 Par exemple: L'Alphabétisation des travailleurs étrangers (Catani M., 1973, Tema); Ethnologie, nspécial, «Travailleurs immigrés»(1977/3); Les Travailleurs étrangers en France est utilisé comme titre dans deux ouvrages (Calame P. et P., 1972, Éditions ouvrières; Minces J., 1973, Seuil). Le travailleur est parfois spécifié par sa nationalité: Le Retour des travailleurs portugais (Poinard M., 1979, La Documentation française); L'Espace des travailleurs tunisiens en France (Simon G., 1979, thèse de géographie).

8 Créée en 1972 l'association a discrètement pris en compte les changements en devenant en 1997 le Groupe d'information et de soutien des immigrés. 
lorsque l'on traite de sa famille et de ceux qu’on appellera la deuxième génération, l'immigré reste perçu avant tout comme un travailleur.'

Un rapport de la Direction de la Populations et des Migrations (Lebon, Marangé, 1982) va amorcer un tournant dans le répertoire des dénominations en associant pour la première fois de façon officielle les termes étranger et immigré à l'origine et à l'ascendance. Jeunes «d'origine étrangère», «descendants d'immigrés», générations «issues de»l'immigration, etc., autant d'expressions dans lesquelles étranger ou immigré sont devenus de purs signifiants de l'extranéité, la réalité qu'il désignent ayant quasiment disparu ou étant en voie de disparaître pour les individus concernés, de plus en plus souvent nés en France et de nationalité française.

Mais comme on l'a déjà signalé, le véritable turning point qui marque l'entrée dans une nouvelle problématique de la question migratoire est la légitimation scientifique et institutionnelle de la préférence accordée à immigré sur étranger comme catégorie pertinente pour la mesure de l'intégration, conduisant à introduire la variable d'origine dans les enquêtes statistiques (Tribalat, 1995 ; HCI I99I). Si la construction de l'immigré comme catégorie statistique dans l'enquête MGIS a sans doute permis, comme le souligne Alexis Spire (1999), de conforter l'idée d'une réussite du processus d’intégration, l’apparition corrélative de l'expression Français de souche, a instauré une auto-référenciation du majoritaire, équivalent de la wasp-ishness décrite par Sarna (1978) pour les États-Unis.

Le terme «travailleur» a fait retour avec l'expression «travailleurs sans papiers » mais la référence au statut de salarié y est subordonnée à l'irrégularité du statut administratif. Les désignations des migrants comme «sans papiers » ou «clandestins » signalent qu'avec l'arrêt de l'immigration et la fermeture des frontières on est passé d'une logique économique à une logique d'ordre public.

9 Par exemple: Questions-réponses sur la scolarisation des enfants de travailleurs migrants (Clevy J., ESF, 1976) ; Vivre dans deux cultures. La condition socio-culturelle des travailleurs migrants et de leurs familles (UNESCO, 1983).

I0 On peut relever que la première est privilégiée par les associations de soutien contre celle d'«immigré clandestin » qui propage une représentation du migrant comme délinquant, fraudeur, hors la loi (Noiriel, 2007, p. 632). 
Une nouvelle fragmentation de la population immigrée entre réguliers et irréguliers s'instaure, soutenue par la rhétorique qui lie le contrôle des flux et l'intégration des étrangers installés, et codifiée par le Droit depuis la loi du i7 juillet $1984^{11}$.

Ces changements d'étiquettes suivent bien sûr l'évolution des flux et des politiques migratoires (arrêt de l'immigration, regroupement familial, fermeture des frontières) mais n'en découlent pas purement et simplement. Ils résultent à la fois de déplacements du sens que connaît un terme dans les usages courants et d'innovations produites par les administrations. Bien qu'elles ne fassent souvent que traduire ces changements d'usages dans un lexique bureaucratique, les désignations et classifications officielles font accepter comme une réalité les catégories qu’elles désignent, ce qu’a souligné Gérard Noiriel (1989) en rappelant que «les jeunes “d'origine immigrée” n'existent pas». Leur pouvoir d'assignation identitaire se trouve pleinement réalisé lorsqu’elles sont validées par des cercles plus larges que les officines bureaucratiques qui les ont fait naître : les associations, les organismes d'études, les médias.

Les catégories ainsi produites ne perdent leurs effets performatifs que lorsque leur définition et leurs limites deviennent explicitement enjeux de lutte, lorsque l'étranger, pour reprendre l'image d’Alfred Schütz (1987, p. 225) «bondit sur la scène » pour parler à la première personne de sa place dans la société qui, du coup, cesse d'être d’accueil.

Quel contenu donner à la catégorie de Français, quels sont les critères qui permettent de reconnaître ceux qui font partie du Nous national, quelles étiquettes sont acceptables ou non pour désigner les majoritaires et les minoritaires? Lorsque les minoritaires s'emparent de ces questions, ils explicitent le changement de leur condition qui n'est plus celle d'étranger / immigré mais celle d'une composante de la société française existant de fait et revendiquant d'y exister de plein droit.

II En même temps qu'elle rompt avec les dispositions précédentes en créant la carte de résident valable 10 ans, la loi maintient ou renforce les dispositifs de rétention ou d'expulsion visant à lutter contre l'immigration clandestine. 
Les stratégies mises en œuvre par les groupes minoritaires ou plutôt leurs porte parole pour revendiquer leur inclusion dans le Nous français ouvrent un vaste domaine d'étude qui reste à explorer ${ }^{12}$. Mais elles impliquent toujours une bataille sur les dénominations.

«Beur » représente un cas intéressant de tentative de construire une identité de groupe revendiquant son intégration sur la base d'une catégorisation globalisante imposée de l'extérieur (arabe). Reprendre en les détournant les nominations données par le dominant peut être une façon de les dénaturaliser en mettant en lumière leur caractère arbitraire. Mais devenu ambigu à force d'être trop consensuel, le terme a fini par être fortement récusé par ceux qui l’avaient (supposément) inventé. Certaines étiquettes peuvent se négocier, comme celle de «Noir» que des mouvements comme le CRAN (Conseil Représentatif des Associations Noires, créé en 2005) ont réussi, avec le soutien d'intellectuels et de personnalités politiques, à imposer comme une identité légitime dans l'espace public; d'autres, comme «Indigènes » créée par le MIR (Mouvement des Indigènes de la République, créé la même année que le CRAN) sont des armes de combat qui ne sont pas destinées à s'imposer, mais à mettre en exergue les fondements (coloniaux) de la domination.

\section{LANGAGE DE LA RACE VS LANGAGE DE LA CULTURE Nouvelle façon de dire l'assimilation?}

Ces auto-désignations (Beurs, Noirs, Indigènes) qui représentent un phénomène nouveau dans la société française réagissent et contribuent au processus de banalisation des références à la race et à l'origine comme déterminants des relations sociales et des identités, que la plupart des chercheurs s'accordent à constater (Noiriel, 2007 ; Fassin, 20ıо ; Poiret, 201 I ; Amselle, 20I I). La généralisation dans les années 1980 de l'expression «deuxième génération » qui pour la première fois dans l'histoire de l'immigration marque les enfants des immigrés de la référence indélébile à leur origine, a représenté la première phase de ce processus. Les

12 Voir pour de premières explorations de cette question: Ndiaye, 2008; Poiret, 2011; Streiff-Fénart, 2012. 
politiques anti-discriminatoires mises en place depuis les années 2000 attestent l'existence de traitements inégaux en fonction de l'ascendance ou de la couleur de la peau, tandis que les phénomènes de pauvreté et d'exclusion sociale ont de plus en plus tendance à être naturalisés par la référence à l'origine des personnes, comme cela s'est manifesté au grand jour lors des émeutes de 2005.

Pour rendre compte de ces phénomènes, deux termes se sont imposés : ethnicisation et racialisation, sans que la différence entre eux soit clairement établie dans les définitions ni dans les usages. Cette zone d’ombre mérite qu’on y regarde de plus près en examinant comment deux auteurs, Jean Loup Amselle (20I I) et Didier Fassin (20ı), qui accordent un privilège exclusif à l'un de ces termes (ethnicisation pour Amselle et racialisation pour Fassin), font fonctionner ces notions.

Les deux auteurs établissent une relation entre le phénomène qu'ils nomment ethnicisation ou racialisation et le recul de l'interprétation des rapports sociaux comme des rapports de classe : le passage du registre de l'exploitation au registre de l'exclusion pour Amselle, le déplacement de la question sociale à la question raciale pour Fassin.

Amselle désigne par ethnicisation le contre-racisme érigé par des entrepreneurs d'identité contre le racisme d’Etat qu'il définit comme le découpage du peuple en fragments donnant naissance à des «communautés de souffrance » (de genre, de race, de culture). Dans sa défense de l'universalisme républicain, il renvoie dos à dos multiculturalisme et racisme comme deux principes symétriques de fragmentation du corps social. Sa dénonciation de «l'ethnicisation de la France » comme un processus d’auto-réalisation des groupes, met sur le même plan l'assignation à une place infériorisée et les luttes pour la reconnaissance, ce qui revient à ne voir dans ces dernières que des impostures : la création artificielle de communautés fictives (les Noirs) par des leaders ethniques auto-proclamés ou des stratégies de diversion manipulées par le pouvoir pour masquer les inégalités socio-économiques. 
Ce sont précisément ces deux dimensions de l'assignation catégorielle et de la reconnaissance que Fassin cherche à mettre en relation à travers la notion de racialisation. Celle-ci désigne à la fois un processus et une problématisation du monde social qui peut se manifester dans les faits de discrimination, dans l'usage des désignations raciales ou ethniques ou dans les affirmations d'identités spécifiques. Ce nouveau concept, s'il présente d’indéniables avantages par rapport à ceux plus anciens de race (en mettant l'accent sur la dimension constructiviste) et de racisme (en évitant la dimension morale de la dénonciation), laisse toutefois entière la question de savoir ce qu'on entend par « racial». Fassin englobe sous ce terme toutes les formes de radicalisation de l'altérité, aboutissant ainsi à la définition de la racialisation comme «l'imposition d'une catégorie explicitement ou implicitement raciale sur des individus et des groupes, généralement pour les dominer ou les exploiter, pour les exclure ou les combattre ». Mais cette définition ne revient-elle pas à repousser d'un cran sans le lever, le risque de circulation tautologique qui conduit à définir comme «racial» ce que le chercheur estime relever de la «racialisation»?

C’est ainsi que dans un autre texte co-signé avec Éric Fassin, le même auteur illustre l'idée que la France est traversée par une «ligne de couleur» (Fassin et Fassin, 2006, p. 5) ou qu'elle est entrée dans «une nouvelle ère d'évidence raciale presquaveuglante» (ibid., p. 7) par des extraits de discours («prolétariat de l'extérieur », «populations à peine françaises », «polygamie» «appartements bondés ») qui font plus référence à une altérité de classe, nationale ou culturelle qu’à la race.

On peut certes mettre au compte de l'euphémisation la variabilité des termes utilisés pour «banaliser le référentiel racial, qu'il soit ou non explicite » (ibid. p. 9). Mais on peut aussi s'intéresser justement à l'explicite, c'est à dire aux répertoires dans lesquels puisent les imputations d’altérité.

On pourra alors constater que la référence explicite à la visibilité de l’apparence comme signe de l'appartenance à une race apparaît constitutive d'un discours et d'une politique de la diversité qui visent à accroître la représentation des «minorités visibles » dans les sphères d'influence, les médias ou les partis politiques. Le label «issu de la diversité » est également au cœur d’une stratégie managériale qui prône «des entreprises aux couleurs de la France» (titre du 
rapport remis au Premier Ministre par C. Bébéar en 2004). Le mot «diversité » évoque dans tous les cas une politique correctrice de discriminations moralement illégitimes et socialement et économiquement dommageables, qui écartent des individus des positions de prestige ou de pouvoir auxquelles ils pourraient prétendre, à partir de leur apparence physique ou de leur ascendance.

La référence culturelle est, quant à elle, mobilisée pour appréhender l'altérité qui reste associée à l'immigration, en provenance des anciennes colonies et plus récemment de la frontière orientale de l'Europe. Si l'apparence y joue un rôle, ne serait-ce que comme indice d'identification, elle est redoublée par la stigmatisation de comportements inappropriés : le mode de vie du nomade, le déficit de socialité de l'habitant de banlieue, la tradition patriarcale ou l'excès de religiosité du musulman.

On peut alors penser que la recomposition des frontières de la société française qu’observent, avec d’autres, à la fois Fassin et Amselle ne revient pas purement et simplement à un déplacement de la problématique de l'immigration à celle de la racialisation pas plus quelle ne se peut se résoudre à un repli généralisé sur des identités culturelles particularistes. Elle consiste plutôt à tracer une nouvelle frontière morale qui relie ou sépare les Nous des Eux en fonction de deux registres de l'altérité : une altérité positive qui célèbre la prise en compte de la diversité et se dit dans l'idiome de la race (les minorités visibles) et une altérité négative sur laquelle porte le soupçon de «communautarisme» et qui se dit dans l'idiome culturel (rapports hommes / femmes, normes éducatives) ou civilisationnel (tel qu'il est dramatisé par l'opposition islam / laïcité dans les polémiques publiques).

Les deux discours se légitiment par la référence aux valeurs républicaines. On retrouve ici une caractéristique proprement française du rapport à l'Autre que Michèle Lamont (2000) a relevé dans son enquête auprès des travailleurs français. Elle observe que la polarisation Nous-Eux est plus marquée avec la population nord-africaine quavec les Noirs (en fait les Antillais), parce qu'elle est redoublée pour les premiers par la différence religieuse et le statut d'immigré et aussi par les reproches qu'on leur fait de refuser de s'intégrer à la société française et d'en respecter les normes de civilité. Elle voit dans les façons différenciées qu'ont les travailleurs français de s'exprimer sur leurs concitoyens noirs ou maghrébins un effet direct du républicanisme qui censure les réfé- 
rences à la couleur de la peau comme base de différenciation, mais encourage la dévalorisation des comportements contraires au code républicain.

Dans les politiques et les discours publics, cette dualité se manifeste toutefois plutôt comme deux principes de réglage des frontières Nous-Eux que par des attitudes différenciées envers des groupes «raciaux» ou «ethniques» définis en substance. L'idiome de la minorité visible peut s’appliquer à des individus qui ne sont pas considérés comme «noirs » et le registre culturel de l'altérisation peut être employé pour stigmatiser les modes de vie des habitants «africains» des banlieues. ${ }^{13}$

Il s’agit bien de deux langages différenciés qui structurent une morale de l’altérité : l'un qui parle de l'inégalité des positions qui touche des individus marqués par un stigmate (la couleur, la «visibilité», le nom), s'impose en dehors de leur volonté et les empêche de réaliser leurs aspirations légitimes à occuper la place qui leur revient dans la société ; et l'autre qui parle de la différence dans des valeurs et des modes de vie, en les considérant au mieux comme la trace de traditions pré-modernes, au pire comme une coupable complaisance à cultiver des particularismes culturels contraires à l'ordre républicain.

La nouveauté ne serait alors pas tant la conversion d'une logique universaliste à une logique différentialiste, qu'une reconfiguration des frontières autour d'une ligne de partage entre deux types d'alters : ceux à qui aucun défaut d'assimilation culturelle ne peut être imputé et qui peuvent revendiquer leur inclusion en tant qu'Autres dans le Nous national, et ceux porteurs de traits culturels ou comportementaux incompatibles avec la société française et qui n'ont d'autre choix que le refoulement vers sa marge ou la dissolution dans le Nous national selon la voie classique de l'assimilation individuelle.

Loin de changer le «modèle $»^{14}$ de l'assimilation, la recomposition des frontières Nous-Eux et des catégories par lesquelles on pense l'altérité serait au contraire, dans cette hypothèse, ce qui permet d'en sauver l'essentiel en adaptant aux réalités du jour la vieille opposition entre assimilables et non assimilables.

13 Et le racisme peut, cela va sans dire, toucher les minoritaires quel que soit l'idiome employé pour désigner leur différence.

14 Sur les «modèles» d'intégration des immigrés en Europe et leur supposée «crise», voir Bertossi et al., 2012. 


\section{CONCLUSION}

On a tenté dans cet article de comprendre comment le concept d'assimilation qui, comme le disait Max Weber à propos de l'ethnie «se volatilise lorsqu'on tente de le cerner avec précision » (1995, p. 139), a représenté un cadre cognitif qui a durablement, quoique inégalement selon les périodes, informé les représentations sociales et les conceptualisations sociologiques de l'immigration.

La notion d'assimilation a fait l'objet de critiques réitérées, dénonçant sa compromission avec l'entreprise coloniale dans le cas de la France ou le mouvement nativiste aux États-Unis, et l’accusant de façon générale de viser l’annihilation des cultures et des identités minoritaires. En dépit de ces critiques, elle a conservé son pouvoir de figuration des rapports entre immigrés et société d’accueil au prix de quelques réaménagements. Mais il ne suffit pas de changer le mot ou d'en rénover la signification pour se débarrasser de l'idée.

En France, le terme «intégration » qu'on a tenté de lui substituer pour atténuer les exigences de conformité trop lourdes qu'elle fait peser sur les groupes minoritaires, a fini, comme tous les euphémismes, par être rattrapé par la signification qu'il cherchait à écarter. «Intégration» en est rapidement venu à charrier la même idée d'un nécessaire alignement des valeurs et des comportements des outsiders, appréhendés comme des groupes spécifiques, à ceux supposés en vigueur dans une société perçue comme homogène et intégrée.

Aux États-Unis, les efforts pour renouveler le sens de la notion d'assimilation en la débarrassant de ses indésirables connotations normatives se sont multipliés au cours des dernières années. Alejandro Portes et Min Zhou (1993), avec les notions de segmented ou downward assimilation, tentent d'en renouveler le sens pour tenir compte des données empiriques qui ne démontrent guère une mobilité sociale et une adhésion à la culture mainstream de la part des nouvelles secondes générations. Rogers Brubaker (200I) soutient que le concept d'assimilation débarrassé de sa visée assimilationniste est indispensable parce qu'il permet de poser des questions sur les degrés de similarité et les différences persistantes entre des populations d'origine immigrée et des populations de référence. Mais, outre qu’on peut se demander pourquoi conserver le signifiant si le signifié n’a plus rien à voir 
avec l'ancien contenu de la définition, on peut relever que ces questions sont en elles-mêmes au fondement de la visée normative de l'assimilation.

On proposera ici l'hypothèse que cette présence têtue d'une notion disqualifiée mais toujours résurgente tient à deux ordres de confusion. Le premier se manifeste dans la difficulté à distinguer clairement son intérêt comme outil théorique des jugements normatifs sur son usage dans le champ politique. Il est significatif qu'un auteur comme Brubaker (200Ib), qui a fourni une contribution décisive pour déconstruire l'autre notion confuse qu'est l'identité en séparant ses usages comme catégorie analytique et comme catégorie de la pratique, tente de réhabiliter la notion d’assimilation en liant entre elles les nouvelles significations qu'elle aurait acquises à la fois dans les discours public et dans la recherche.

Une autre confusion qui la rend «bonne à penser» (et en fait du même coup un mauvais concept scientifique) est qu'elle fonctionne, diraient les ethnométhodologues, comme thème et ressource, à la fois comme objet d'enquête sociologique et comme idée d'arrière plan qui informe nos façons de penser tout à la fois l'immigré comme étranger à la Nation, les groupes comme des porteurs de culture, et la culture comme héritage et propriété.

Pour lever cet impensé, plusieurs pistes de recherche méritent d’être explorées conjointement. Le développement depuis les années 1990 d'une problématique en terme de mobilités transnationales ou de «territoires circulatoires » (Glick Schiller et al., 1992; Tarrius, 1992) a ouvert la voie à une compréhension des activités sociales des immigrés libérée du carcan du «nationalisme méthodologique ». Les réfutations par les anthropologues des conceptions essentialistes des ethnies ont contribué à faire de la culture une catégorie émergente dans un espace de relations inter-groupes (Amselle et M’Bokolo, 1985 ; Barth in Poutignat et Streiff-Fénart, 1995). L’ethnométhodologie a par ailleurs fourni des contributions trop méconnues: celle de Michael Moerman (1988) montrant comment les attributions ethniques sont des accomplissements qui se donnent à voir dans l'interaction, ou de Wes Sharrock (1974) démontrant par l'absurde les implicites qui font d'un stock de connaissance culturel une propriété d'un groupe. ${ }^{15}$ 
Bien que puisant à des traditions de recherche fort différentes, chacune de ces approches contribue à sa manière à mettre en évidence les présupposés inhérents à la notion d'assimilation. On peut penser qu'au-delà de la question migratoire, le croisement des pistes de recherche qu'elles suggèrent pourrait contribuer à une réflexion d’intérêt plus général sur les formes émergentes d’identité / altérité dans les sociétés contemporaines.

\section{BIBLIOGRAPHIE}

ALBA, R. D., NEE V., 1997, «Rethinking Assimilation Theory for a New Era of Immigration », International Migration Review, 31-4, p. 826-874.

AMSELLE J.L., E. M’BOKOLO (dir.), 1985, Au cœur de l'ethnie, Paris, La Découverte.

AMSELLE J. L., 1996, Vers un multiculturalisme français. L'empire de la coutume, Paris, Aubier.

-, 2011, L'Ethnicisation de la France, Paris, Lignes.

BERTOSSI C., DUYVENDAK J. W., M. A. SCHAIN (eds.), 2012, Comparative European Politics, special issue, «The Problems with National Models of Integration: A Franco-Dutch Comparison», |0-3.

BORGOGNO V., 1988, «L'insertion urbaine des immigrés: cadre de compréhension et scénarios possibles d'avenir», in Prospective culturelle, Actes du colloque européen, Marseille, 1987, p. 159-178.

BORIE W.D., 1959, The Cultural Integration of Immigrants, UNESCO.

BRUBAKER R., 1997, Citoyenneté et nationalité en France et en Allemagne, Paris, Belin.

-, 200।, «The Return of Assimilation? Changing perspectives on immigration and its sequels in France, Germany, and the United States», Ethnic and Racial Studies, 24-4, p. 531-548.

-, 200 lb, «Au-delà de l'identité», Actes de la recherche en sciences sociales, 139-4 p. 66-85.

DE RUDDER V., GUILLON M., 1987, Autochtones et immigrés en quartier populaire. D’Aligre à l'ïlot Chalon, Paris, L'Harmattan. 
DESCLOITRES R., 1967, Les Travailleurs étrangers. Leur adaptation au travail et à la vie urbaine, Paris, OCDE.

ESCAFRÉ-DUBLET A., 201।, «Mesurer l'intégration des immigrés. Genèse et histoire des enquêtes Girard Stoezel, 1945-1953», Genèses, 84-3, p. 93-II2.

FASSIN D., 20I0, «Ni race ni racisme. Ce que racialiser veut dire», in Les Nouvelles Frontières de la société française, D. Fassin (éd.), Paris, La Découverte. -, FASSIN É. (éds), 2006, De la question sociale à la question raciale? Paris, La Découverte.

FAVELL A., 200I, «Integration policy and integration research in Europe: a review and critique», in Citizenship Today: Global Perspectives and Practices, T. A. Aleinikoff \& D. Klusmeyer (eds), Washington DC, Brooking Institute/Carnegie Endowment for International Peace, p. 349-399.

GLAZER N., MOYNIHAN D.P. (eds), 1963, Beyond the Melting-Pot, Cambridge Mass., Harvard University Press.

-, 1975, Ethnicity, Theory and Experience, Cambridge Mass., Harvard University Press.

GIRARD A., 1954, «L'assimilation culturelle des immigrants en Belgique», Population, 9- I, p. 146-148.

GLICK SHILLER N., BASH L., BLANC-SZANTON C. (eds.), 1992, Towards a Transnational Perspective on Migration: Race, Class, Ethnicity and Nationalism Reconsidered, New York, New York Academy of Sciences.

GORDON M., 1964, Assimilation in American life, the Role of Race, Religion, and National Origins. New York, Oxford University Press.

GRANOTIER B., 1970, Les Travailleurs immigrés en France, Paris, Maspéro.

GUILLON M., TABOADA-LEONETTI I., 1986, Le Triangle de Choisy, un quartier chinois à Paris, Paris, L'Harmattan.

HAJJAT A., 2010, «Port du hijab et "défaut d'assimilation”. Étude d'un cas problématique pour l'acquisition de la nationalité française», Sociologie, 4-I, p. 439-455.

HAUT CONSEIL À L'INTÉGRATION, I99I, Pour un modèle français d'intégration, Paris, La Documentation française (collection des rapports officiels). 
-, 1995, Liens culturels et Intégration, Rapport au Premier Ministre, Paris,

La Documentation française (collection des rapports officiels).

HOVANESSIAN M., 1992, Le Lien communautaire. Trois générations d'Arméniens, Paris, Armand Colin.

HUGHES E.C., MAC GILL H.H., 1952, Where people meet. Racial and ethnic frontiers, Wesport Connecticut, Grenwwod Press Publishers. The Free Press.

JOHNSON C. S., 1922, The Negro in Chicago. A study of race relations and a race riot, Chicago, The University of Chicago Press.

LAGRANGE H., 2010, Le Déni des cultures, Paris, Seuil.

LAMONT M., 2000, The Dignity of Working Men: Morality and the Boundaries of Race, Class, and Immigration, Cambridge Mass.,Harvard University Press.

LEBON A., MARANGÉ J.,1982, L'Insertion des jeunes d'origine étrangère dans la société française, Paris, La Documentation Française.

LORCERIE F., 1999, «Les habits neufs de l'assimilation en France», in Migrations internationales et relations interethniques. Recherche, politique et société, I. Simon-Barouh et V. De Rudder, (éds), Paris, L’Harmattan, p. 297-344.

MERCIER C., 1977, Les Déracinés du capital, Lyon, Presses Universitaires.

MAUCO G., 1932, Les Étrangers en France. Étude géographique sur leur rôle dans l'activité économique, Paris, Armand Colin.

MEILLASSOUX C., 1976, Femmes, greniers et capitaux. Paris, Maspéro.

MOERMAN M., 1988, Talking Culture, Philadelphia, University of Pensylvania Press.

NDIAYE P., 2008, La Condition noire. Essai sur une minorité française, Paris, Calmann-Lévy.

NOIRIEL G., 2007, Immigration, antisémitisme et racisme en France, Paris, Fayard.

-, 1989, «Les jeunes "d'origine immigrée" n'existent pas», in Les Politiques d'intégration des jeunes issus de l'immigration, B. Lorreyte (éd.), Paris, L'Harmattan, p. 21 I-22I.

ORIOL M. (dir.), 1984, Les Variations de l'identité, Rapport final de l'ATP CNRS 054. PAON M., 1926, L'Immigration en France, Paris, Payot. 
PARK R. E., 1926, «Our Racial Frontier on the Pacific», in Race and Culture, R. E. Park, Glencoe, The Free Press, 1950, p. I50-I5I.

PATTERSON S., 1963, Dark strangers: a sociological study of the absorption of a recent West Indian migrant group in Brixton, South London, London, Tavistock Publications.

PERRIAUX A. S., VARRO G., 1991, «Les sens d'une catégorisation: les OS immigrés», Langage et Société, 58, p.5-36.

POIRET Ch., 20II, «Les processus d'ethnicisation et de raci(ali)sation dans la France contemporaine: Africains, Ultramarins et "Noirs"》, Revue Européenne des Migrations Internationales, 27-I, p. 107-127.

PORTES A., ZHOU M., 1993, «The New Second Generation: Segmented Assimilation and its Variants $\gg$, Annals of the American Academy of Political and Social Sciences, 530, p.74-96.

POUTIGNAT Ph., 1994, «La problématique de l'ethnicité: du groupe ethnique à l'organisation sociale des différences culturelles», in Cultures ouvertes, sociétés interculturelles: du contact à l'interaction, C. Labat, G. Vermes (éds), Paris, L'Harmattan, p. |47-161.

-, STREIFF-FÉNART J., 1995, Théories de l'ethnicité (suivi de Les groupes ethniques et leurs frontières par Fredrik Barth), Paris, PUF.

RAVENSTEIN E. G., 1889, «The Laws of Migration», Journal of the Royal Statistical Society, LII-2, p. 24I-305.

ROEDIGER D. R., 199I, The Wages of Whitness, London, New York, Verso.

SARNA D. J., 1978, From Immigrants to Ethnics: Toward a New Theory of Ethnicization, Ethnicity, 5, p. 370-378.

SAYAD A., 1981, Le Phénomène migratoire: une relation de domination, Annuaire de l'Afrique du Nord, XX, p. 365-406.

SCHÜTZ A., 1987 (1944), «L'Étranger. Essai de psychologie sociale», in Le Chercheur et le Quotidien, Paris, Méridiens Klincksieck.

SHARROCK W.W., 1974, «On Owning Knowledge», in Ethnomethodology, Roy Turner (ed.) Harmondsworth, Penguin Books, p. 45-53. 
SPIRE A., 1999, «De l'étranger à l'immigré», Actes de la Recherche en Sciences Sociales, 129, p. 50-56.

STREIFF-FÉNART J., 1994, Ruser avec la tradition. Le mariage dans les familles maghrébines en France, Projet, 239, p. 57-61.

-, 2012, «A French Dilemma: Anti-Discrimination Policies and Minority Claims in Contemporary France», Comparative European Politics, 10-3, p. 283-300.

TARRIUS A., 1992, Les Fourmis d'Europe, Paris, L'Harmattan.

THOMAS W.I., ZNANIECKI F., 1918-1920, The polish Peasant in Europe and America: monography of an immigrant group, Boston, Richard G. Badger, The Gorham Press.

TODD E., 1994, Le Destin des immigrés. Assimilation et ségrégation dans les démocraties occidentales, Paris, Seuil.

TRIBALAT M., 1995, Faire France. Une enquête sur les immigrés et leurs enfants, Paris, La Découverte.

TRIPIER M., 1987, L'Immigration dans la classe ouvrière en France, Thèse de doctorat, Université de Nantes.

WARNER W. L., SROLE L., 1945, The social Systems of American Ethnic Groups, New Haven, Yale University Press.

WIMMER A., 2008, The Making and Unmaking of Ethnic Boundaries: a Multilevel Process Theory, American Journal of Sociology, 113-4, p. 970-1022.

-, GLICK SCHILLER N., 2003, «Methodological nationalism, the social science and the study of migration: An essay in historical epistemology», The International Migration Review 37-3, p.576-610.

WEBER M., 1995 (1922), Économie et Société - 2, Paris, Plon Pocket.

ZOLBERG, A., WOON L. L., 1999, «Why Islam is like Spanish: Cultural Incorporation in Europe and the United States», Politics and Society, 27-I, p. 5-38. 
